\title{
Germanica
}

\section{Im Zeichen des (geschichtlichen) Verbrechens. Zum Phänomen der deutschsprachigen Doku- Krimis}

Sous le signe du crime historique. À propos du phénomène du roman policier documentaire allemand

Under the Sign of (Historical) Crime. On the Phenomenon of German Documental Crime Novel

\section{Wolfgang Brylla}

\section{OpenEdition \\ Journals}

Édition électronique

URL : http://journals.openedition.org/germanica/3191

DOI : 10.4000/germanica.3191

ISSN : 2107-0784

\section{Éditeur}

Université de Lille

\section{Édition imprimée}

Date de publication : 30 septembre 2016

Pagination : 97-109

ISBN : 9782913857377

ISSN : 0984-2632

\section{Référence électronique}

Wolfgang Brylla, «Im Zeichen des (geschichtlichen) Verbrechens. Zum Phänomen der deutschsprachigen Doku-Krimis », Germanica [Online], 58 | 2016, Online erschienen am: 30 September 2018, abgerufen am 06 Oktober 2020. URL : http://journals.openedition.org/germanica/3191 ; DOI : https://doi.org/10.4000/germanica.3191 


\section{Le polar juridique, historique et documentaire}





\title{
Im Zeichen des (geschichtlichen) Verbrechens. Zum Phänomen der deutschsprachigen Doku-Krimis
}

\author{
Wolfgang BRYLLA \\ Universität Zielona Góra (Polen)
}

\section{Die (Krimi-)Geschichte boomt}

In den letzten zwei Jahrzehnten ist ein stets wachsendes Interesse an Geschichte und Geschichtsaufarbeitung festzustellen. Die nicht mehr vorhandene Vergangenheit, an die man sich erinnern muss, damit sie vor dem Vergessen bewahrt wird, wurde zu einem der wichtigsten Themen der Popkultur, was dazu führte, dass sich mehrere Medien der Historie im Sinne vom Stoff bedient hatten. Barbara Korte und Sylvia Paletschek sprechen im Zusammenhang mit dem zu konstatierenden Geschichtsboom von ,popkulturellen Repräsentationen“ der Geschichte1, die eine „Geschichtskultur“ auslösten². Die ins Zentrum der medialen Betrachtung gerückte historische Welt des Faktischen und

1. - Barbara Korte/ Sylvia Paletschek, „Geschichte in populären Medien und Genres: Vom historischen Roman zum Computerspiel”, in Barbara Korte / Sylvia Paletschek (Hrsg.), History Goes Pop. Zur Repräsentationen von Geschichte in populären Medien und Genres, Bielefeld, transcript, 2009, S. 9-60, hier S. 9.

2. - Ebd., S. 10. 
des Realen resp. des Geschehenen ist für die Literatur kein Neuland, das man erst betritt ${ }^{3}$. Nach Jahren der Missbilligung und scharfer Kritik, in denen der historische Roman mit dem „Odium“ der Obsoletheit und des Alten versehen wurde ${ }^{4}$, feiert das Genre heutzutage ein großes Comeback, eine Renaissance 5 .

Dem aktuellen und andauernden Trend, eine Art fiktiv-literarische Geschichtsschreibung zu betreiben, folgte auch der Kriminalroman, der trotz seiner gattungskonstituierenden Korsett-Struktur auf Variabilität angewiesen ist ${ }^{6}$. Bis dato war die Krimiliteratur, in erster Linie in ihrer klassischen Ausprägung, eher ahistorisch determiniert ${ }^{7}$. Geschichte bildete somit einen ,blinden Fleck ${ }^{6}$. Erst in den 1990er Jahren, als erste historische Kriminalromane des russischen Autors Boris Akunin veröffentlicht wurden, wandte sich das Blatt, obwohl schon vorher Vorläufer wie Umberto Eco mit dem Verweis auf den historischen Background versuchten, dem Krimi einen neuen Touch zu verpassen ${ }^{9}$. Die Entwicklung des historischen Krimiplots ging so rasend schnell vonstatten, dass man heute den Eindruck gewinnen könnte, das Krimigenre habe quasi ein Vorrecht auf die Benutzung und Problematisierung der Geschichte ${ }^{10}$. Die in den Krimis präsen-

3. - Spätestens seit Walter Scotts Ritterromanen etablierte sich in der Literaturforschung die Gattung des historischen Romans, der in Deutschland sich vor allem Ende des 19. und Anfang des 20. Jahrhunderts hoher Beliebtheit erfreute. Alfred Döblin meinte sogar in seinem berühmten Plädoyer für den Geschichtsroman, dass er einerseits zum Symbol und andererseits zum Synonym des Romans schlechthin wurde (Alfred Döblin, „Der historische Roman und wir“, in Alfred Döblin, Schriften zu Ästhetik, Poetik und Literatur, Olten/ Freiburg im Breisgau, Walter Verlag, 1989, S. 291-316).

4. - Ansgar Nünning, ,,Von der fiktionalisierten Historie zur metahistoriographischen Fiktion: Bausteine für eine narratologische und funktionsgeschichtliche Theorie, Typologie und Geschichte des postmodernen historischen Romans“, in Daniel Fulda/ Silvia Serena Tschopp (Hrsg.), Literatur und Geschichte. Ein Kompendium zu ihrem Verhältnis von der Aufklärung bis zur Gegenwart, Berlin, De Gruyter, 2002, S. 541570, hier S. 542.

5. - Siehe: Ulrich Kittstein, „Mit Geschichte will man etwas“. Historisches Erzählen in der Weimarer Republik und im Exil (1918-1945), Würzburg, Königshausen \& Neumann, 2006, S. 9.

6. - Vgl. Bertolt Brecht, „Über die Popularität des Kriminalromans“, in Jochen Vogt (Hrsg.), Der Kriminalroman. Poetik - Theorie - Geschichte, München, W. Fink, 1998, S. 33-37.

7. - Vgl. Ulrich Schulz-Buschhaus, „Leonardo Sciascia oder Die Beunruhigung des Kriminalromanlesers", in Erhard Schütz (Hrsg.), Zur Aktualität des Kriminalromans, München, W. Fink 1978, S. 142-154, hier S. 143.

8. - Klaus Inderthal, ,Selbstgemachte Notwendigkeit. Zur Geschichte und Theorie einer populären Prosa: Detektiv- und Kriminalliteratur", in Erhard Schütz (Hrsg.): Zur Aktualität des Kriminalromans, München, W. Fink, 1978, S. 20-57, hier S. 52.

9. - Siehe: Ulrich Suerbaum, Krimi. Eine Analyse der Gattung, Stuttgart, Reclam, 1984, S. 206-211.

10. - Achim Saupe, Der Historiker als Detektiv - der Detektiv als Historiker. 
tierte Historie ist jedoch mehr oder weniger eine ,fiktive Historizität ${ }^{‘ 11}$. Verwende man den Ausdruck von Paul Ricoeur, könnte auch die Rede sein von der ,Historisierung der Fiktion“' ${ }^{‘}$.

\section{Erzählen vom wirklichen Verbrechen: Pitaval}

Schaut man dezidierter auf die Subgattung des historischen Kriminalromans, fallen seine weiteren Subvarianten ins Auge. $\mathrm{Zu}$ divergieren wäre bspw. der Antike-Krimi, der Mittelalter-Krimi oder der Retro-Krimi. In der Zwischenzeit setzte sich jedoch auch eine andere Variable, ein anderes Narrativ, durch, das man im deutschsprachigen Raum meistens als Doku-Krimi etikettiert. Zum Hauptvertreter dieses Genres wurde Horst Bosetzky, der z.B. in Wie ein Tier. Der S-Bahn-Mörder (1995), Der kalte Engel (2002) und Die Bestie vom

Historik, Kriminalistik und der Nationalsozialismus als Kriminalroman, Bielefeld, transcript, 2009, S. 12. Der Historiker Wojciech Burszta, einer der wichtigsten polnischen Literaturforscher, hat den Hang des zeitgenössischen Kriminalromans zur Thematisierung und Konzentration auf das Vergangene als „Flucht in die Geschichte“ umschrieben (Wojciech Burszta, „Bronię kryminału!“, in: Polityka Nr. 11/ 2005 vom 19. März 2005, Sonderheft „Niezbędnik Inteligenta”), Mariusz Czubaj bezeichnete diese Affinität als „Sturz in die Zeit“ (Mariusz Czubaj, Etnolog w Mieście Grzechu. Powieść kryminalna jako świadectwo antropologiczne, Gdańsk, Oficynka, 2010, S. 13). Dabei machen Korte/ Paletschek darauf aufmerksam, dass das Phänomen des Geschichtskrimis nicht nur mit der Rückwendung auf historisches Denken zu erklären ist. Sie differenzieren zwischen zwei Klassen des historisch orientierten Kriminalromans. Zum einen heben Korte/ Paletschek solche Krimis hervor, deren Verbrechenshandlungen sich durch einen Gegenwartsbezug legitimieren, der allerdings auf die Geschichte zurückverweist. Zum anderen erscheinen solche Krimitexte, deren erzählte Geschichte, literarische Figuren und beschriebene Räume der Kriminalität nur in der Vergangenheit angesiedelt sind (Barbara Korte/ Sylvia Paletschek, „Geschichte und Kriminalgeschichte(n): Texte, Kontexte, Zugänge“, in Barbara Korte/ Sylvia Paletschek (Hrsg.), Geschichte im Krimi. Beiträge aus den Kulturwissenschaften, Köln/ Weimar/ Wien, Böhlau, 2009, S. 7-27, hier 10).

11. - Siehe: Tom Zwaenepoel, Dem guten Wahrheitsfinder auf der Spur. Das populäre Krimigenre in der Literatur und im ZDF-Fernsehen, Würzburg, Königshausen \& Neumann, 2004, S. 56.

12. - Paul Ricoeur, Zeit und Erzählung III, München. W. Fink, 1991, S. 294-311. Solche auf historischem (Vor-) Wissen gegründeten Kriminalromane können zwar mehr über die Geschichte aussagen als der ,normale ' historische Roman (vgl. Ray B. Browne/ Lawrence A. Kreisler, ,Introduction“, in Ray B. Browne/ Lawrence A. Kreisler (Hrsg.), The Detective as Historian. History and Art in Historical Crime Fiction, Madison, The University of Wisconsin Press, S. 1-9, hier S. 5), sie sind allerdings stets aus dem Blickwinkel der Fiktionalität aufzufassen und der möglichen „Interdependenzen“ (Saupe, Der Historiker als Detektiv, a.a.O., S. 26) zwischen belegter Geschichtsschreibung und gedichteter Krimifiktion. Deswegen nimmt es kaum wunder, dass die meisten Geschichtskrimis nur auf die historischen Schlüsselereignisse, die dem Mainstream zugänglich und bekannt sind, rekurrieren; sie transportieren, stabilisieren und bestätigen somit das „sichere Wissen“ der Leserschaft, das nicht hinterfragt werden muss (Korte/ Paletschek, „Geschichte und Kriminalgeschichte(n)“, a.a.O, 2009, S. 10). 
Schlesischen Bahnhof (2004) jeweils eine andere wirtschaftliche und politische Zeitepoche in der deutschen Geschichte umreißt, dokumentierte Kriminalfälle als Fallgeschichten darstellt und somit zwischen den Zeilen den Anspruch auf Authentizität und Wahrheit erhebt. Bosetzky (Pseudonym -ky) rekonstruiert in allen drei Krimis auf einer durch das Doku-Material fundierten Grundlage die Mordfälle aufs Neue; sie werden literarisiert und als literarisch-wissenschaftliche Arbeit vermarktet. Im Folgenden soll das narrative Sujet der Romane von -ky ebenso wie die Gesamtkomposition seiner Krimi-Texte in den Fokus geraten, die, so könnte man zumindest vermuten, eine „Aporetik der [vergangenen] Zeitlichkeit" hätten suggerieren können ${ }^{13}$.

Die Beschreibung und Wiederaufnahme von ,echten' kriminellen Tatstrafen, Morden, Totschlaggeschichten etc. zum Zweck und im Dienst der Literatur ist keine Eigenschaft der Moderne, sondern ein ästhetisches Konzept aus dem 18. und 19. Jahrhundert, als wahre Kriminalfälle anhand von Ermittlungs- und Gerichtsverhandlungsakten, Verhörprotokollen oder Tätergeständnissen literarisiert und dem Lesepublikum als Bücher bzw. Berichte serviert wurden. In den Pitaval-Sammlungen ${ }^{14}$ wurden jedoch keine Deduktionsprozesse in Gang gesetzt, die für die Detektivliteratur der ersten Stunde typisch waren: Es gibt zwar ein Verbrechen, aber dieses wird nicht als Problem und Geheimnis, das man entziffern muss, geschildert, sondern schon als ein gelöster Fall ${ }^{15}$. Die Konvention dieser Art von Literatur beschränkte sich, wie Jörg Schönert mit Blick auf die deutsche Kriminalgeschichte bemerkt, auf sensationell-unterhaltende, erbaulich-moralisierende und räsonierend-informative Funktionen ${ }^{16}$. Mit der Herauskristallisierung der Detektiverzählung verloren die Fallgeschichten zunächst an Relevanz, es kam zu einer Umakzentuierung und Schwerpunktverlegung von

13. - Zum Problem der „Aporetik der Zeitlichkeit“ siehe: Ricoeur, Zeit und Erzählung III, a.a.O.

14. - Peter Nusser, Der Kriminalroman, Stuttgart, J.B. Metzler, 2003, S. 75 f.

15. - Die Pitaval-Erzählungen leben nicht von der kompositorischen Rhetorik des Detektivromans, dem das narrative Gerüst von 1) Verbrechen, 2) Untersuchung und 3) Aufdeckung zugrunde liegt (vgl. ebd., S. 22). Auch eine linear-rückgreifende Erzählhaltung (vgl. Stanko Lasić, Poetyka powieści kryminalnej, Warszawa, PIW, 1976, S. 56) ist dem Pitaval völlig fremd.

16. - Jörg Schönert, „Kriminalgeschichten in der deutschen Literatur zwischen 1770 und 1890. Zur Entwicklung des Genres in sozialgeschichtlicher Perspektive“, in Jochen Vogt (Hrsg.), Der Kriminalroman. Poetik - Theorie - Geschichte, München, W. Fink, 1998, S. 322-349, hier S. 326. Im Laufe der Zeit veränderten der Pitaval und andere Verbrechensdarstellungen ihre Grundform und pendelten zwischen „,aktenmäßiger Behandlung' authentischer Fälle [...] und ,Kriminovellen“ im Sinne von freier literarischer Bearbeitung konkreter Fälle oder Fiktionen von Kriminalfällen und ihrer Strafverfolgung" (ebd., S. 327-328). 
plausibler Realitätsbebilderung zum deduktiven Spiel, dessen Hauptziel das Rätsellösen war ${ }^{17}$.

Die Dokumentarkrimis von Bosetzky scheinen sich in die Tradition der Pitavale einzuschreiben, in denen weniger die Fahndung nach dem Missetäter von Belang ist, weil er schon sozusagen von der Geschichte entlarvt und zu einer historischen Figur, die häufig auch Kultstatus erreichte, hochstilisiert wurde. Eine gravierendere Rolle kommt bei Bosetzky vielmehr dem historisch-psychologisch-sozialen Hintergrund zu. Dem klassischen whodunit? wird ein Riegel vorgeschoben; nicht die Fragen nach dem Wer? oder nach dem Wie? stehen im Mittelpunkt, sondern die Warum-Frage. Warum wurden die Menschen zu Massenmördern? Hatten die Zeitgeschichte, das Milieu oder das familiäre Umfeld dem Charakterprofil der Gewaltverbrecher ihren Stempel aufgedrückt? Die Hauptachse der Romane wurde nicht um die TäterFiguren, sondern vielmehr um die Menschen, die sich hinter den Tätern verbergen, aufgebaut.

Betrachtet man die oben genannten Kriminalromane nur oberflächlich, würde man sie ohne lange zu zögern als gängige Kriminalliteratur bezeichnen. Auf den Plan tritt ein Schwerverbrecher, der wie beim Thriller schon im Voraus ein Gesicht und einen Namen besitzt. Die Leser werden deshalb nicht zu einem Ratespiel eingeladen, sie werden mit einer polizeilichen Fahndungsprozedur konfrontiert und zur Observierung der kräftezehrenden, oft fehler- und lückenhaften ins Nichts hinauslaufenden Ermittlung gezwungen. Mit anderen Worten: man beobachtet die Ermittler, wie sie einen historisch wahren Fall lösen und wie sie den Straftäter schnappen. Spannung resultiert somit nicht aus der Häufung von Aha-Effekten, sondern aus dem Gegebenen und der Nachvollziehung der (literarischen) Fakten, die man mit der Geschichtsschreibung hätte vergleichen können. Das für die Detektivliteratur charakteristische System von Fragen und Antworten 18 wird umgewandelt in ein System von Wissen und Beobachten. Vor dieser Folie wird auch der Satz von Richard Alewyn ${ }^{19}$, nach dem der Erzähler alles und der Leser nichts wisse, umgemodelt. Nicht nur der Erzähler weiß alles, auch die Leserschaft und die Geschichte wissen alles. Als einziger Unwissender erscheint, was paradox klingen mag, der Krimi selbst.

17. - Siehe: Suerbaum, Krimi, a.a.O., S. 30-34.

18. - Ebd., S. 22.

19. - Richard Alewyn, ,Anatomie des Detektivromans“, in Jochen Vogt (Hrsg.), Der Kriminalroman. Poetik - Theorie - Geschichte, München, W. Fink, 1998, S. 52-72, hier S. 54. 


\section{Ein Denkmal für den Frauenschänder Großmann: Die Bestie vom Schlesischen Bahnhof}

Wie offenbart sich diese Unwissenheit des dokumentarischen Kriminalromans von Bosetzky? Formell und erzähltechnisch gesehen wurde Die Bestie vom Schlesischen Bahnhof als sechsteiliger Roman mit zusätzlichem Prolog, Epilog und Anhang entworfen. Schon im Vorspiel erfährt man den Namen des Frauenmörders. Es ist der Wurstverkäufer Karl Großmann, der immer wieder Frauen vom Bahnhof abschleppt, die Anfang der 1920er Jahre in die Weltstadt Berlin aus ihren Provinznestern kamen, um sich aus Armut, Dreck und Chancenlosigkeit $\mathrm{zu}$ befreien. Großmann bietet den jungen Frauen eine Unterkunft und eine Stelle als Hausmädchen an. Bosetzkys allwissender Er-Erzähler ${ }^{20}$, denn er verfügt über eine Unmenge an Informationen, die Großmanns (geistes-)gestörten Lebensweg als auch die Erzählgegenwart betreffen, widmet dem Wiederaufrollen der Kindheit Großmanns die ersten zwei Teile seines Romans. Skizziert und in gewisser Hinsicht erörtert werden soll der mit Schlachtwerkzeug operierende Mördermechanismus bzw. -körper Großmanns, den die soziale Umwelt mehr oder weniger zur kaltblütigen Tötungsmaschine gemacht hatte. Für solche Banditen wie Großmann ist Einfühlungsvermögen nur ein bedeutungsloses Wort, das nicht zum Werte- und Ideologiehorizont eines Frauenmörders und -vergewaltigers gehört. Der Erzähler bemüht sich darum, den Grund für die sexuelle und psychische Anomalie Großmanns in seiner Kinderund Jugendzeit zu suchen, als er in den Wäldern Neuruppins Karnickel ritualmäßig hingerichtet hatte. Schon als Minderjähriger verging er sich mehrmals an gleichaltrigen Mädchen, wanderte ins Zuchthaus, aber die Erfahrung des Gefängnisses hat, statt ihn zu heilen, den Defekt nicht nur verschlimmert, sondern ließ auch bestimmte Verteidigungs- bzw. Entschuldigungsstrategien entstehen. So schob Großmann bewusst die Schuld für sein Tierwerden - die Animalisierung - auf seine Eltern und die ihn umgegebene Natur:

Was kann ich dafür. [...] Es steckte nun mal so drinne in mir, und ich kann nichts dagegen machen. Das ist die Natur. So ist die nu mal. Da kann man doch 'n Menschen nich für verurteilen, dass er so is. Det is doch nicht seine Schuld. Sperren Sie Gott ins Zuchthaus oder meine Eltern, die sind schuld an allet ${ }^{21}$.

20. - Die narratologische Analyse stützt sich vor allem auf: Matias Martinez/ Michael Scheffel, Einführung in die Erzähltheorie, München, C.H. Beck, 2005.

21. - Horst Bosetzky, Die Bestie vom Schlesischen Bahnhof, Berlin, Jaron, 2004, S. 59. 
Vom kranken Trieb geleitet nahm Großmann seine Verbrechen nicht als Sünden und Gräueltaten wahr, sondern als Projektion seiner Seele, ohne die er nicht hätte überleben können. Somit musste er die unschuldigen Frauen misshandeln und töten, um sich selbst zu behaupten und sich vor seiner persönlichen Geschichte zu rechtfertigen. Obwohl die Polizei ihn schon seit längerem im Visier hatte und die Nachbarn aus der Mietskaserne sich über Gestank von verwesenen Leichenteilen aus seiner Wohnung beklagten, kam es niemandem in den Sinn, dass der Lumpensammler Großmann ein Sadist und hemmungsloser Mörder sein könnte. Ein ähnlicher Eindruck wird auch vom Erzähler erzeugt, der stets mithilfe von Analepsen auf die Kindheit von Großmann zu sprechen kommt und im späteren Handlungsverlauf, wenn Großmann sich der Polizei stellen muss, auch auf Verhörprotokolle oder Fachbeurteilungen von Psychologen zurückgreift. Die polizeiliche Ermittlung nach dem Schlachter vom Schlesischen Bahnhof beginnt erst im vierten Teil des Romans, der Leser hatte bis zu diesem Zeitpunkt die Gelegenheit, sich mit dem vom Erzähler angebotenen komplexen Konstitutionsbild von Großmann vertraut zu machen. Seine Übeltaten werden in die Zeitgeschichte integriert und vor der historischen Kulisse der ersten Nachkriegsjahre beleuchtet. Die ersten Subkapitel fangen meistens mit einer kurzen historischen Einführung an, aus der die Leser Kenntnis von der politischen Stimmung in der Weimarer Republik, der Wirtschaftskrise und der steigenden Kriminalitätsrate erhalten. Großmanns Frauenmordserie reiht sich in diese Misslage ein, seine Taten sind keine Taten mehr, sondern ein Sinnbild der Zeit, sogar eine „Kunst“22, wie Großmann selbst glaubt. Der Strafprozess und die Verhandlung, die im letzten Teil gestützt durch Aussagen der herangezogenen Psychiater illustriert werden, verstärken dieses äußere Erscheinungsbild. Großmann wird als ,schwer belastete[r] Mann“, bei dem „Defekte des ethischen Verhaltens“ zu erkennen sind, oder als „epileptoider Imbeziller" mit ,erethischer Hemmungslosigkeit“" gegeiBelt ${ }^{23}$. Noch vor der Urteilssprechung erhängt sich Großmann in seiner Zelle mithilfe eines Bettlakens.

Anhand von Quellennachweisen und Fachliteratur, die sehr oft zitiert werden, spürt Bosetzky der Tätergeschichte von Großmann nach. Da aber die Story für den Schriftsteller so drastisch und unmenschlich war, musste er sie mit einer "Love-Story und viel ablenkender Handlung“ aufpeppen, wie er selbst im Nachwort bekennt ${ }^{24}$. Die erzählerische Konstruktion von Die Bestie vom Schlesischen Bahnhof verdeutlicht die Ablenkungstaktik des Erzählers/ Autors. Der Doku-Krimi wirkt zu

22. - Ebd., S. 241.

23. - Ebd., S. 279 und S. 283.

24. - Ebd., S. 316. 
durchkombiniert. Das Switchen zwischen wissenschaftlicher Recherche und erfundener Krimihandlung mag im Hinblick auf die Textstruktur interessant und vielversprechend sein, im Falle der GroßmannGeschichte führt sie lediglich dazu, dass man auf der einen Seite einen Lustmörder zu Gesicht bekommt, der für seine Taten hätte büßen und verteufelt werden sollen, auf der anderen Seite hegt man für ihn auch Sympathie, Verständnis und Mitleid. Im Epilog belauscht der heterodiegetische Erzähler ein Gespräch zwischen den fiktiven Figuren Zeztscha und Tschau, die sich über Großmann unterhalten. Das Fazit ist erstaunlich und bedrückend zugleich:

Die Leute gieren ja geradezu nach solchen Geschichten, denn in vielen von uns, den Männern insbesondere, steckt ein Stückchen Mörder - und Menschen wie Karl Großmann begehen ihre Verbrechen auch stellvertretend für andere. Also: Ein Karl-Großmann-Denkmal wäre wirklich eine Attraktion für die deutsche Hauptstadt ${ }^{25}$.

Der historische Roman von Bosetzky, den man in Anspielung auf die Typologie von Ansgar Nünning als historiographische Fiktion bezeichnen kann $^{26}$, setzt, indem er eben hybridisch die Gattung des Krimis mit dem Geschichtssachbuch vermengt ${ }^{27}$, Großmann gewollt oder ungewollt ein Denkmal, das der Triebmörder nicht verdient. In Die Bestie vom Schlesischen Bahnhof werden fast alle geläufigen narrativen Krimitechniken und -modelle über den Haufen geworfen. Deswegen sollte statt des Terminus Doku-Krimi die Bezeichnung Krimi-Doku zur Anwendung kommen, um das Hybride des Romans hervorzustreichen.

25. - Ebd., S. 289.

26. - Vgl. Nünning, ,Von der fiktionalisierten Historie zur metahistoriographischen Fiktion“, a.a.O., S. 547-548. Nünning divergiert zwischen fünf Kategorien des historischen Romans. In seinem System tauchen auf: der dokumentarische historische Roman, der realistische historische Roman, der revisionistische historische Roman, der metahistorische Roman und die metahistoriographische Fiktion. Die ersten beiden Romantypen zeichnen sich durch eine große Dichte an Realitätsreferenzen aus. Sowohl der revisionistische als auch der metahistorische Roman sowie die metahistoriographische Fiktion charakterisieren sich mehr oder weniger durch die Hinterfragung der Geschichtsschreibung. Bei Bosetzky bekommt man es mit keiner dieser Textklassen zu tun. Statt dessen tritt eine ,historiographische Fiktion " hervor, die sich der Geschichtsschreibung im großen Grad bedienend und auf Dokumente beziehend weniger auf den Einsatz und die Produktion von Realitätsreferenzen baut, sondern die Erschaffung einer historisierenden, historiographischen Textwelt im Sinne hat, die man nicht in Zweifel ziehen kann.

27. - Auch der historische Roman sei unter dem Gesichtspunkt der Hybridität (vgl. Hans Vilmar Geppert, Der historische Roman. Geschichte umerzählt - von Walter Scott bis zur Gegenwart, Tübingen, Francke Verlag, 2009, S. 9). 


\section{Der Krimi wird selbst zum Opfer: Wie ein Tier. Der S-Bahn-Mörder}

Das Stichwort Hybride gilt auch für die beiden anderen PseudoKrimis von Bosetzky. Sogar der Autor selbst macht keinen Hehl daraus, dass er divergente Genres verwoben hatte, um Spannungselemente zu erzeugen. Im Nachwort zu Wie ein Tier. Der S-Bahn-Mörder - erneut wird die Tier-Metapher gebraucht - gesteht Bosetzky Folgendes:

Zugleich aber ist mein S-Bahn-Mörderbuch auch ein Roman, eben Produkt meiner Phantasie und Ergebnis diverser Kunstgriffe aus dem Spannungsschreibergewerbe. Da war auf cliffhanger hinzuarbeiten, und thrill und suspense waren ebenso einzuweben wie die Muster des klassischen Polizeiromans [... ${ }^{28}$.

Wie ein Tier, in dem die Geschichte über Paul Ogorzow erzählt wird, der in den 1940er Jahren in der Berliner S-Bahn oder in der Nähe des Bahnhofsknotens Rummelsburg ahnungslose Frauen schändete, basiert ebenso wie der Großmann-Roman auf Fachliteratur, die in Form von Zitaten oder abgebildeten Zeitungsausschnitten in die Haupthandlung eingeflossen sind. Trotzdem wird kein totaler Dokumentarismus wie im Falle von Die Bestie vom Schlesischen Bahnhof angepeilt, sondern eher eine authentische Fiktionalität mit implizierten historischen Realitätsaufnahmen, die durch Wirklichkeitsreferenzen strukturiert ist, die Bosetzky „true crime quickie“ nennt ${ }^{29}$. Bei dieser Verhistorisierung sei er nach einem ,archäologischen Prinzip“30 verfahren, indem er Akten einsah, in die Zeitepoche eintauchte und wissenschaftliche Studien zurate zog.

Bevor der Erzähler Bosetzky detaillierter die historische Figur des Frauenhassers und Lustmörders Ogorzow exemplifiziert - sie tritt mit Namen und ihrem Portofolio erst im dritten der insgesamt fünf Teile unter dem Titel Der ganz normale Alltag eines Mörders auf - weiß er die Kriegsstadt Berlin in Szene zu setzen, die in Verdunkelung vor der Bombardierungsgefahr versinkt. Die Verdunkelungspflicht, die den Einwohnern Sicherheit geben sollte, entpuppt sich für den Täter Ogorzow, der als Fahrkartenkontrolleur bei der S-Bahn arbeitet, bei seinen geplanten oder impulsiv durchgeführten Frauenangriffen als sehr hilfreich ${ }^{31}$. In der Dunkelheit kann man sich verstecken, die Dunkelheit verdeckt die Identität des Mörders, die Dunkelheit schenkt

28. - Horst Bosetzky, Wie ein Tier. Der S-Bahn-Mörder, München, dtv, 2009, S. 321.

29. - Ebd., S. 323.

30. - Ebd., S. 319.

31. - Saupe, Der Historiker als Detektiv, a.a.O., S. 412. 
Anonymität und animiert zu Gewalt. Auch das Krimi-Narrativ mit dessen Erzählkonstruktion scheint sich in der Dunkelheit verirrt zu haben. Eingeleitet wird die Haupthandlung von der omnipräsenten Erzähler-Stimme, die alle Fäden zieht und nach Belieben den Fortgang der Geschichte steuern kann, mit der Schilderung von frauenfeindlichen Überfällen im nationalsozialistischen Berlin. Obwohl der Hauptermittler Baronna ziemlich schnell einen Fahrkartenkontrolleur verdächtigt und obwohl die Tatwaffe gefunden wird - ein Bleikabel -, wird die erste Hypothese fallen gelassen. Die NS-Polizei tappt im Dunkeln, wobei die Ermittlungsansätze, was sich am Ende zeigen wird, korrekt und schlüssig waren:

„Bleiben zwei Möglichkeiten... [...] Der Täter ist sich einer Sache furchtbar sicher. Er setzt einmal darauf, daß in den Zeiten der Verdunkelung alles möglich ist, und zum anderen traut er der Kriminalpolizei sehr wenig zu. Wegen der personellen Unterbesetzung, alle Männer an der Front, bei der SS oder sonst wo gebunden.“ „Trauen Sie ihm so viel Intelligenz denn zu?“ „Intelligenz nicht, aber Instinkt. So wie Raubtiere ihn haben. [...] Er tut es eben, dumpf und triebhaft“32.

Die Krimihandlung dümpelt dahin, nur durch narrative CrossDressing-Handgriffe wird sie halbwegs am Leben erhalten ${ }^{33}$. So wird von der Berliner Polizei ein Undercover-Agent unter die S-Bahn-Arbeiter eingeschleust, Baronna und seine Polizeikollegin Behrens werden zwischenzeitlich zu Lockvögeln. Das Ergebnis des Cross-Dressings ist die Mobilisierung und Verzerrung von schematischen Krimi-Strukturen sowie „binäre[n] Differenzen“: „Der Gejagte wird zum Jäger, der Verbrecher zum Staatsdiener und umgekehrt" ${ }^{34}$. Die Umdifferenzierung hat zur Folge, dass aus der Täterstelle eine Leerstelle wird ${ }^{35}$. Dasselbe gilt allerdings auch für die Detektiv-Stelle, da Baronna später an die Ostfront ziehen und sich am Völkermord beteiligen muss. Im Plot Wie ein Tier finden sich demnach ausschließlich Opferfiguren, auch der Krimitext selbst ist als solch eine zu sehen. Schon wieder werden nicht nur die Erzählstruktur, sondern auch der Handlungsvorgang diffus.

\section{Verfangen in der Ich-Bezogenheit: Der kalte Engel}

Als vollständige ,wissenschaftliche Arbeit“ soll dahingegen Bosetzkys Der kalte Engel nicht aufgefasst werden, da der Autor aus

32. - Bosetzky, Wie ein Tier, a.a.O., S. 97.

33. - Irina Gradinari, Genre, Gender und Lustmord. Mörderische Geschlechterfantasien der deutschsprachigen Gegenwartsprosa, Bielefeld, transcript, 2011, S. 289.

34. - Ebd.

35. - Ebd., S. 310. 
„dramaturgischen Gründen“ in dem „true crime“ etwas „hinzuerfunden" habe ${ }^{36}$. Trotzdem rundete Bosetzky seinen Roman, in dem der Fall von Elisabeth Kusian bearbeitet wird, die im geteilten Berlin nach 1945, in dieser „Trümmerwüste“ wie es heißt ${ }^{37}$, Menschen liquidiert und sie dann genau wie Großmann zerstückelt, mit einem umfassenden Literaturverzeichnis auf. Vom Erzählaufbau und der Erzählsyntax her ähnelt Der kalte Engel der Großmann-Geschichte insoweit, als zum Schluss der Strafprozess der Kusian wiedergegeben wird. Die vorherigen Kapitel steuern eben auf das Gerichtsverfahren zu, dem das ganze Erzählgefüge untergeordnet ist. Da die Mordserie über die innerdeutsche Grenze hinausgeht, wird ein Ermittlerteam auf den Fall angesetzt, das sich aus einer ostdeutschen Polizistin und einem westdeutschen Kommissar zusammensetzt. In der krisenhaften Nachkriegszeit, noch vor dem Wirtschaftwunder, als das alltägliche Leben von Armut und Hunger bestimmt war, ist die Erinnerung an das Dritte Reich immer noch aktuell. Dem Anschein nach hat sich nach der Potsdamer Konferenz alles verändert, in Wirklichkeit jedoch ist das nicht der Fall, wie es der Er-Erzähler, der einen Zugang zum Innenleben der Figuren hat, unter Hinweis auf Bacheran diagnostiziert: „Das neue Deutschland, fand Bacheran, war auch nach der Entnazifizierung weithin das alte geblieben. Ein Anzug blieb ein Anzug, auch wenn man ihn umgefärbt hatte, von braun auf rot oder auf schwarz" ${ }^{38}$.

Dem Anzug, der Uniform wird im Roman eine wesentliche Funktion beigemessen, denn auch die weibliche Mörderin Elisabeth Kusian trägt eine Uniform, genauer gesagt einen weißen Krankenschwesterkittel. Wegen ihrer Position in der Nachkriegsgesellschaft genießt sie ein hohes Ansehen: „Es kann nicht sein, was nicht sein darf, das heißt, bestimmte BerufsgruppendürfenkeineMördersein, sonstbrichtdas ganzeWertgefüge zusammen: Ärzte, Pfarrer, Mütter, Krankenschwestern, mütterliche Krankenschwestern“39. Bei Bosetzky kommt es zu einer semantischen Rekonfiguration. Eben diese Rekalibrierung löst nicht nur bei den Lesern, sondern auch bei den Zeugen und Prozessbeobachtern Entsetzen aus. Niemand würde je daran glauben, dass eine Krankenschwester andere Menschen umbringen könnte. Auch Kusian ist sich dessen sicher: „NIE WÜRDE KRANKENSCHWESTER EINEN MORD BEGEHEN!"“40 vermerkt sie in ihren Ich-Ausführungen, die man auch als undatiertes Tagebuch auslegen könnte. In den Ogorzow- und Großmann-Romanen meldeten sich die Täter zwar auch in der Ich-Form zu Wort, aber nur

36. - Horst Bosetzky, Der kalte Engel, Berlin, Jaron, 2002, S. 363.

37. - Ebd., S. 7.

38. - Ebd., S. 88.

39. - Ebd., S. 295.

40. - Ebd., S. 179. 
in einem Vernehmungsprotokoll oder offiziellen Geständnis, das dem Dokumentenarchiv von Bosetzky entnommen wurde. Bei der KusianStory wird solch ein faktuelles Bekennerschreiben fiktionalisiert und mithilfe eines narrativen Perspektivenwechsels in die Haupthandlung integriert. Der dritte Teil des Romans Ich. Ich. Ich. Elisabeth Kusian beinhaltet die persönliche Erzählung von Kusian, die dem fiktiven Außenrahmen der geführten polizeilichen West-Ost-Ermittlung erst einen Sinn gibt. In dem Bekenntnis wird nicht mehr die Sichtweise des Erzählers, ausgestattet mit einer Null-Fokalisierung, vorgezogen, sondern die homodiegetische intime Ich-Erzählgrammatik. Erst durch diese Vertrautheit und Figurennähe wird man sich der Unfassbarkeit der Mordtaten von Kusian bewusst. Zum Vorschein kommt ein psychisches Selbstporträt einer fragilen Persönlichkeit, deren mentale Biographie durch das Kriegsgeschehen gestört und auf den Kopf gestellt wurde ${ }^{41}$.

Die Fahnder sind nicht imstande, den Sachverhalt aufzuklären, Kusian zählt zwar zum Kreis der Verdächtigen, aber in Wirklichkeit wird ihr der zweifache Mord nicht zugetraut. Sie selbst geht sogar aufs Revier, um ihre Aussage zu der getöteten SchreibmaschinenVerkäuferin zu tätigen. Erst als sich die Indizien gegen Kusian bestätigen, die Beweislast immer größer wird und sie dem Druck sowie der Erpressung, man werde ihren Ex-Mann wegen Mittäterschaft anklagen, nicht standhält, zerbricht ihre zementierte kalte Fassade. Kusian gibt die Morde zu, wird verurteilt, stirbt sieben Jahre später in der Haft an einem Krebsleiden, wie Bosetzky, und nicht dessen Erzähler, unter Rückgriff auf dokumentierte Unterlagen anführt. Die finale Übersetzung der Handlung von der Erzähl- auf die Ich-Autorenebene unterstreicht nur, welches Anliegen Bosetzky wohl vorschwebte: nämlich einen Dokumentarroman, eine Zeitstudie, zu verfassen, die mit der Gattung Kriminalroman außer dem Figurenpersonal und dem Motiv des Verbrechens wenig Gemeinsamkeiten hat.

\section{Fazit: ,Unwissende' Krimis}

Horst Bosetzkys Doku-Krimis, wobei man auf diese Gattungskategorisierung verzichten sollte, behandeln historische Verbrechen, historische Täter und historische Gerichtsverhandlungen aus den 1920er, 30er und 40er Jahren. In dieser Hinsicht spielen die fiktionalisierten, literarisierten Verbrechenshandlungen vor einer historisch nachweisbaren Dekoration; sie sind somit echt in ihrer wissen-

41. - „Wäre sie auch zur Doppelmörderin geworden, wenn es kein Drittes Reich gegeben hätte, sondern sich Deutschland zu einer ganz normalen Republik entwickelt hätte, wie die USA etwa? Nein. Oder doch?“ (ebd., S. 326). Diese Frage stellt sich zumindest Bacheran, auf die er keine konkrete Antwort parat hat. 
schaftlichen Dogmatik. Gleichzeitig sind sie allerdings fiktiv bezüglich ihres literarischen Potentials. Die Verbindung von Geschichtsschreibung und (Kriminal-)Literatur rief bei Bosetzky eine textliche Erschließung der Mentalitätsgeschichte der Verbrecher-Typen aus der deutschen Geschichte hervor. Es handelt sich in diesem Punkt um keine gattungsdefinierten Krimis, obwohl in den Romanen auch Detektive und andere Vertreter der ingroup-Konstellation ${ }^{42}$ in Erscheinung treten. Die Polizei strebt eine Ermittlung an mit dem Ziel, die Täter zu überführen, das Rätsel zu lösen und die Schuldigen zu bestrafen. Aber im Endeffekt fällt dieser krimibezogene Handlungszweig bei Bosetzkys Doku-Verbrechensschilderungen kaum ins Gewicht. Auch ohne den Einsatz von solchen und ähnlichen für das Krimigenre eigentümlichen Strukturkomponenten würden die Romane im Rahmen der Literarizität im Allgemeinen ,funktionieren'. Statt von Doku-Krimis zu sprechen, sollte man Bosetzkys Großerzählungen jedoch vielmehr als historische Romane deklarieren, die grauenhafte geschichtliche Delikte zum Gegenstand haben. Aus diesem Grund könnte man den Ausdruck, neue (historische) Pitavale ins Gespräch bringen, in denen eine wirkliche Verbrechergeschichte versinnbildlicht wird, die aber einem ,Feintuning ${ }^{6}$ (fiktive Figuren, räumliche Ausschmückung, Erzählweise) unterzogen wurde und als historiographic fiction interpretiert werden könnte. Der Zusatz crime, von dem jedoch Bosetzky selbst immer wieder Gebrauch macht, wirkt in dieser Typologie deplatziert: die Mörder werden nicht vom Detektiv entlarvt, die Auflösung ist quasi a priori von der Geschichte vorgegeben. Bosetzky liefert bloß eine literarisierte Geschichtschronik des Verbrechens, was ihm zwar gutzuschreiben ist. Aber Krimis sind die Kusian-, Großmann- und Ogorzow-Stories nicht. Oder anders: die sogenannten Doku-Krimis sind ,unwissende‘ Krimis. 
